In the last four decades there has been enormous progress in the treatment for childhood cancer; the present 5-year survival rate is estimated to be $80 \%$. The increased population of cancer survivors gives us the possibility to assess life quality as well as the late effects of anticancer treatment. In multicentre studies, it has been shown that $60 \%$ of survivors have at least one late effect, and one third experience severe or lifethreatening sequelae. Survivors of childhood cancers are at risk of recurrence of primary malignancy, development of second cancer and chronic disease of the respiratory and cardiovascular system, which increase the mortality rate. Other late complications, such as diseases affecting kidneys, digestive system or bones, and hormonal and metabolic disturbances lead to deterioration of life quality. This report presents treatment-related factors that increase mortality of cancer survivors by contributing to the development of second cancers, cardiotoxicity or pulmonary complications.

Key words: children, cancer survivors, late effects, second cancers, cardiotoxicity, lung toxicity, late mortality.

\section{Late mortality of long-term survivors of childhood cancer}

\section{Maryna Krawczuk-Rybak}

Department of Paediatric Oncology and Haematology, Medical University of Bialystok

The significant progress observed over the last 40 years in the diagnostics and treatment for cancer in children has caused an improvement in 5-year event-free survival (EFS) up to 80\%. Before 1970, anticancer therapy involved mainly radiotherapy and surgery. Later, chemotherapy began to play a role. In subsequent years, the chemotherapy programmes were modified and so were the radiotherapy and surgery programmes. Both single-centre and multicentre studies on the health condition of long-term survivors of childhood cancer have focused on the causes of premature deaths of patients treated for cancer in childhood. Until 1970, the major cause of mortality was recurrence of primary tumour (over 70\%). Research conducted in recent years has demonstrated the highest mortality rate among patients treated for CNS cancer (11-15\%), leukaemia (12-14\%) and bone tumours (10-16\%), and the lowest for retinoblastoma (2-5\%) and Wilms tumour (3-4\%) [1].

\section{Epidemiology}

As shown by epidemiological data, in the USA one out of 640 young adults aged 20-39 years is a long-term survivor of childhood cancer. Fairly representative groups of several or even tens of thousands of childhood cancer survivors have been observed since 1940 in the United States, Canada, Germany, Great Britain and Italy [2-4]. This allows for a multidirectional analysis of health condition, organ function and life quality of an increasing number of survivors, and promotes the changes in radiotherapy and chemotherapy techniques.

The largest multidirectional investigations carried out in the United States as part of the Childhood Cancer Survivor Study (CCSS) in a group of over 20 thousand survivors indicate that more than $60 \%$ of them have a chronic disorder and $27.5 \%$ have a severe life-threatening disease [5]. Of the group of 20,483 patients diagnosed in the years 1970-1986, 2821 died. The most common cause of death was progression or recurrence of primary or second cancer (standardized mortality ratio $S M R=15.2)$, circulatory disorders $(S M R=$ $=7.0)$ and respiratory diseases $(S M R=8.8)$. The total SMR was estimated to be 10.8 [6].

According to the data published by the CCSS in 2009, as compared to the previous years, there was no increase in mortality rate due to recurrence or progression of primary tumour; however, the mortality rate due to second cancers and late organ complications was higher. A higher mortality rate is observed among women (SMR $=13.2$ ) as compared to men $(S M R=6.7)$, due to second cancers, circulatory diseases and other causes. The standardized mortality rate also depends on the type of primary tumour. It is the highest in patients after treatment for medulloblastoma $(S M R=23.4)$, Hodgkin lymphoma $(S M R=20.0)$ and Ewing's sarcoma $(S M R=20.0)$, which is mainly associated with the occurrence of second cancer following radiotherapy. However, the highest mortality rate due to heart diseases is observed in patients treated for kidney tumours $(S M R=12.7)$ and Hodgkin lymphoma $(S M R=11.9)$ 
with anthracyclines and radiotherapy of the thorax. Patients who underwent treatment for acute myeloblastic leukaemia and neuroblastoma have the highest SMR due to respiratory diseases (SMR = 24.9 and 11.4, respectively). Other factors contributing to high SMR include age at diagnosis (0-4 years of life), application of radiotherapy, alkylating drugs and high doses of epipodophyllotoxins [6-8].

The analysis conducted by the BCCSS (British CCSS) on a group of almost 18 thousand patients treated for cancer under the age of 15 in the years 1940-1991 estimated the SMR to be 10.7. Like in the American study, the SMR decreased along with time of follow-up, but even 45 years after diagnosis it was threefold higher than expected. The absolute excess risk (AER, i.e. the number of observed cases minus the expected cases/person/year) of deaths due to primary tumour recurrence was reduced with the time elapsed since diagnosis, but it increased when second tumours and circulatory disorders developed [3]. Scandinavian studies conducted among 13,711 survivors aged under 20 years at the time of diagnosis, treated in the years 1960-1989, showed a similar SMR (10.8), though higher due to primary tumour recurrence. However, SMR due to second cancer was estimated to be 4.9 [9]. In a study carried out in Italy, out of 6402 adult survivors of childhood cancer, 890 died, mainly due to recurrence or second malignancy in a group of survivors treated for solid tumours. On the other hand, complications after therapy were the most common cause of deaths in a group of patients treated for leukaemia and lymphomas [4].

\section{Second cancers}

Recent studies indicate that second cancers are the leading cause of preterm deaths in 5-year survivors of childhood cancer. As the population of children cured of cancer increases, also the incidence of second malignancies in this group will grow. The risk of second cancer refers to $3-20 \%$ of patients treated for childhood cancer 20 years before, being 20 -fold higher than in the general population. The risk is related to the type of the primary tumour, treatment applied, genetic factors, age and sex [10-12].

Previously applied radiotherapy is the major cause of increased mortality after anticancer treatment, also due to second malignancy (leukaemia and solid tumours). It contributes to the development of chronic circulatory and pulmonary diseases, obesity and thyroid disorders [13]. The cytostatics that influence the occurrence of second cancers (mainly leukaemias) include alkylating drugs, topoisomerase II inhibitors and epipodophyllotoxins [14].

Cancers affecting the CNS are the most common solid tumours in childhood. The CCSS has shown that $74 \%$ of patients reach 5-year EFS. However, the mortality rate in this group is 13 times higher than in the general population, especially 5-9 years following diagnosis. In the subsequent years, SMR decreases, but even 30-40 years after diagnosis it is still high $(S M R=8.4)$. The main causes of deaths among survivors include recurrence of primary and second cancers, especially those affecting the central nervous system (CNS), thyroid cancers and soft tissue sarcomas. First of all, this is associated with previously applied radiothera- py, its dose in particular. The highest SMR in patients with diagnosed primary tumour is found after treatment for medulloblastoma $(S M R=17.4)$ and ependymoma $(S M R=$ 15.9). The most common second cancers are gliomas and meningiomas $[15,16]$.

Acute leukaemias account for approximately $25 \%$ of childhood cancers, with 5 -year survival averaging $80 \%$. The 15 -year cumulative incidence rate of second cancer is $2.5-4 \%$, whereas the 30 -year rate is approximately $6 \%$. The risk for CNS cancer as the second tumour is 10-20 times higher than in the general population. The most common second cancers are CNS tumours (53\%), leukaemia, lymphoma and skin carcinoma. The risk factors include age below 5 years at diagnosis, female gender, radiation of the CNS and medulla (in the treatment/prevention of leukaemia) and relapse. As after treatment for CNS tumours, gliomas and meningiomas are the most common. The latent period for second cancer depends on the type of malignancy, being approximately 9 years for gliomas to 20 for meningiomas. Radiotherapy dose is an additional risk factor $[17,18]$.

Subsequent acute leukaemia is another second malignancy observed after treatment for acute lymphoblastic leukaemia. Myelodysplastic syndrome (MDS) is also possible. The major pathogenetic factor is exposure to topoisomerase II inhibitors, epipodophyllotoxins and anthracyclines and high doses of alkylating drugs. Second leukaemia develops after treatment with alkylating drugs in older children diagnosed at the age of 10-16 years, often after primary cancer relapse, with a latency of 4-10 years. They can be accompanied by abnormalities in chromosome 5 and 7, frequently preceded by MDS. On the other hand, topoisomerase II inhibitors can induce leukaemia which has a shorter latency (1-3 years) and at an earlier age [10, 14].

Total body irradiation (TBI) used as myeloablative conditioning, previous radio- and chemotherapy, and immunosuppression increase the risk of second cancer in patients after bone marrow transplantation. Also cancers of the haemopoietic system (acute myeloblastic leukaemia/MDS), lymphoproliferative diseases as well as solid tumours of CNS, thyroid or breast are the most common. Factors that increase the risk of second cancer include chronic disease, graft versus host disease (GvHD), TBI application at a younger age, transplantation from an unrelated donor or administration of antithymocyte globulin [19-21].

Neuroblastoma accounts for approximately $6 \%$ of childhood cancers. Rubino et al. in a group of 544 patients treated for neuroblastoma in the years 1948-86 found a second cancer in 12, especially in the third decade of the followup [22]. However, in the CCSS conducted in 954 patients treated for neuroblastoma in the years 1970-86, SMR was 5.6 , with relapse or second cancer being the most frequent cause of death (3.5\% after 20 years and $7 \%$ after 30 years). Metaiodobenzylguanidine 131-I (MIBG-131 I) in combination with radiotherapy increases the risk of second leukaemia, MDS, breast and thyroid cancers [23].

Retinoblastoma, a highly curable cancer (95\%), is hereditary in approximately $40 \%$ of cases, with an accompanying deletion of the suppressor gene Rb-1, which coexists with genetic predisposition to the development of second 
malignancy, including bone sarcoma, soft tissue sarcoma and melanoma. Also higher is the risk of lung cancer, breast cancer, colon cancer, and urinary bladder cancer, especially over the age of 30 years. The cumulative risk for the second malignancy is high (38.2\%), and $60-70 \%$ of second cancers are located in the radiation field. In children diagnosed before the age of 5 years, there is a risk of trilateral retinoblastoma, i.e. a neuroblastic tumour most frequently located in the pineal gland $[10,24]$.

The risk of second cancer after treatment for Hodgkin lymphoma is particularly high due to radiotherapy and chemotherapy. The cumulative incidence in a 25-year follow-up is found to be $19 \%$, although data obtained by many centres represent divergent values depending on the follow-up duration. The most common second malignancies include acute leukaemias, and cancers of the breast, thyroid and gastrointestinal tract, although cancers of the skin, the urinary system, the brain, bone and soft tissue sarcomas are also observed. A younger age at diagnosis (12-16 years), being female, and increased dose of radiotherapy are independent risk factors $[10,25]$. The risk of leukaemia is from 4 to 175 times higher, and the risk of non-Hodgkin lymphoma 5-20 times higher than in the general population of children. This is associated with previous chemotherapy, exposure to alkylating drugs and topoisomerase II inhibitors. Second leukaemia may appear later (after alkylating drugs) or earlier, after approximately 2 years, following administration of topoisomerase II inhibitors. The prognosis is usually serious, with substantial proliferation of cancer cells and poor response to treatment. Solid tumours as second malignancies develop basically at a later age and affect mainly patients who received a high cumulative dose of chemotherapy and radiotherapy $[10,26]$.

The relative risk for breast cancer is 5.2 to 136 times higher, and the cumulative incidence increases with age. Women aged 30-40 years are affected rather than those in their twenties, usually 10-20 years after termination of treatment for Hodgkin lymphoma. This is particularly related to previous radiotherapy and its cumulative dose. Breast cancer is frequently bilateral. A risk factor is age at radiotherapy (puberty/young adults). The use of chemotherapy is controversial: many observations suggest that the administration of alkylating drugs and radiotherapy of the ovarian region lead to their damage and thus decrease the risk of breast cancer. Splenectomy is another risk factor as it impairs the immunological supervision, which promotes proliferation of cancer cells $[10,25,27]$.

Thyroid cancers develop after treatment for Hodgkin lymphoma 1.74-36.4 times more often than in the general population of children; the risk increases 10 years after obtaining remission and is particularly high 40 years after the treatment. Papillary thyroid carcinomas account for $63-90 \%$ of thyroid malignancies. Radiotherapy is the most significant risk factor; $95 \%$ of tumours are located in the field of radiation. Genetic predisposition, environmental factors, immunosuppression and female gender also play an important role. Prognosis is usually good and approximately $80 \%$ achieve long-term remission [25, 26, 28].
The likelihood of cancers of the gastrointestinal tract is 7-22 times higher than in the general population of children, with the oesophagus, stomach and colon being affected. The latent period ranges from 10 to 20 years. The risk factors are age at diagnosis (younger), radiotherapy (increases with dose and radiation field), chemotherapy, gastroesophageal reflux, steroids, and Barrett's oesophagus [25].

After treatment for Hodgkin's lymphoma (10-15 years), the risk of lung cancer and bone and soft tissue sarcomas also increases. Apart from radiotherapy, the risk factors include chemotherapy and immunosuppression, and for lung cancer, smoking [25].

Genetic predisposition is a significant risk factor of second cancer, e.g. family members with Li-Fraumeni syndrome presenting suppressor gene 553 mutations, which in combination with previously applied radiotherapy and chemotherapy increases the risk for second malignancy. Also genetic polymorphism of drug metabolizing/inactivating enzymes, such as thiopurine S-methyltransferase (TPMT), glutathione S-transferase (GST) and CYP3A4, can increase the risk of second cancer, e.g. brain tumour, acute myeloblastic leukaemia or MDS [10].

\section{The respiratory system}

Respiratory complications are the second most common cause of mortality after anticancer treatment in childhood $(S M R=8.8)$, accounting for $2.6 \%$ of deaths [2]. In comparison to healthy relatives, cancer survivors more frequently develop pulmonary fibrosis, recurrent pneumonia, bronchitis, sinusitis, pleural fibrosis and pleuritis, chronic cough, thoracic deformity or effort dyspnoea. Five and more years after the cancer diagnosis, the risk for pulmonary fibrosis increases dramatically, especially after chest radiation and chemotherapy with busulfan, bleomycin, melphalan, lomustine (CCNU) and cyclophosphamide. Twenty years after treatment, pulmonary fibrosis affects $3.5 \%$ of survivors [5]. Progressive pulmonary fibrosis may lead to the development of pulmonary heart and circulatory/respiratory failure, and the necessity to use oxygen therapy. Following lung irradiation, after the acute inflammation phase, fibrosis develops and ventilation becomes gradually impaired [29, 30]. Chest irradiation in children may cause abnormal growth of bony structures and muscles, leading to deformations. Patients whose lung fields were irradiated with a total dose of 12-14 Gy as part of treatment for Wilms tumour and metastases to the lungs demonstrate reduced lung and life capacity. Thoracotomy additionally reduces lung capacity. Among cytostatics, bleomycin is particularly toxic for the lungs. Its administration leads to lung toxicity in approximately $10 \%$ of treated patients. The histopathological picture shows exudates within the pulmonary alveoli, with the subsequent organization, formation of hyaline membranes, interstitial fibrosis, atypical proliferation of alveolar cells and metaplasia [31-33]. Combined treatment of Hodgkin lymphoma, mantle field radiotherapy and particularly toxic chemotherapy (bleomycin), as well as treatment of germcell tumours with bleomycin, put these patients at risk for pulmonary complications. Therapy with alkylating drugs (carmustine, busulfan, melphalan), e.g. in the treatment of 
brain tumours or in a pretransplantation procedure, also contributes to lung fibrosis. Following treatment for acute lymphoblastic leukaemia, there are usually no evident clinical symptoms of respiratory failure; however, symptoms of restrictive lung disease may appear. The symptoms may result from the application of anthracyclines and circulatory failure, CNS radiotherapy and exposure to methotrexate, cytosine arabinoside and cyclophosphamide [34, 35].

Respiratory complications are common in patients who have undergone bone marrow transplantation. This is related to the application of TBI and potentially pulmotoxic drugs (busulfan, melphalan). The application of TBI may cause interstitial pneumonia and obliterative bronchiolitis. Postradiation pneumonia manifestations include cough, dyspnoea, chest pain and subfebrile states. The prevalence depends on the fraction and cumulative doses. Whole lung radiation with a single dose of 8.2 Gy leads to death of $5 \%$ of irradiated patients, while a dose of 9.3 Gy or 11 Gy causes lethal pneumonitis in $50 \%$ or $80 \%$, respectively. Interstitial pneumonia is the cause of approximately $40 \%$ of deaths after transplant. Bronchiolitis obliterans organizing pneumonia (BOOP) accompanies graft versus host disease (GvHD). Chronic obturative lung disease after bone marrow transplant is an extremely severe complication, with a mortality rate of over $70 \%$ [30].

\section{Cardiovascular diseases}

Cardiovascular disorders are also a serious life-threatening complication after anticancer treatment ( $S M R=7.0)$. The cumulative probability of death due to heart disorders increases 15-20 years after the diagnosis. SMR for sudden deaths due to heart disorders is $3.9[36,37]$.

There is a wide range of cardiovascular complications, including dilated cardiomyopathy, myocardial infarction, valvular abnormalities, conduction system dysfunction, coronary and cerebral vascular dysfunction as well as pericarditis $[2,14,35]$.

Anthracyclines, especially doxorubicin, are particularly cardiotoxic cytostatics, which through the toxic action of reactive oxygen species destroy myocytes and the conducting system, thus leading to structural and functional abnormalities in the heart, especially left ventricular dysfunction.

Late cardiotoxicity manifestation depends on age at therapy (most at risk are younger children $<7$ years of life), cumulative dose (> $300 \mathrm{mg} / \mathrm{m}^{2}$ ), gender (female) and time that has passed since treatment. Progressive impaired heart contractility and reduced mass of the left ventricle with increased afterload lead to chronic congestive heart failure (CHF), poorly responding to pharmacotherapy [36-38]. According to the New York Heart Association (NYHA), the mortality rate due to CHF is high (approximately 50\%) [37].

Subclinical late cardiomyopathy or left ventricular dysfunction, clinically asymptomatic, is diagnosed by echocardiography. Its occurrence depends on the cumulative dose of anthracyclines. A study conducted by Lipshultz et al. showed increased afterload or decreased left ventricular contractility in $75 \%$ of survivors of childhood cancer, after the application of doxorubicin at a mean dose of $334 \mathrm{mg} / \mathrm{m}^{2}$ [39].
Long-term effects of subclinical cardiac dysfunction are not well known, although it is suggested to cause an increase in mortality rate due to cardiac disorders, infarcts and strokes [37, 40].

Mediastinal, thoracic and spinal radiotherapy increases twofold the risk of deaths due to cardiac causes. Mantle field radiotherapy used in the treatment of Hodgkin lymphoma leads after 10 years to the occurrence of clinical symptoms of heart disease in approximately 5-6\% of those examined. Ischaemic heart disease, premature coronary atheromatosis, valve destruction, cardiomyopathy, and constricting pericarditis can be observed. Symptomatic ischaemic heart disease develops 9 years after radiation exposure in approximately $10 \%$ of patients, whereas stenosis of cervical arteries and subclavicular artery affects $7.4 \%$ of survivors 17 years after radiotherapy [37].

Irradiation destroys vascular endothelia, thus initiating the atherosclerotic process, which is accelerated by the generation of reactive oxygen species and inflammation in response to endothelial damage. Direct myocyte damage occurs. Left ventricular dysfunction is observed in $10-20 \%$ of people after radiation to the cardiac region with a dose of 30-36 Gy, whereas a dose of 50 Gy leads to a decrease in the ejection fraction in approximately $30 \%$ of patients. Myocardial dysfunction and fibrosis result in restrictive cardiomyopathy. Disturbances in the diastolic fraction are found to predominate in patients treated only with radiotherapy, whereas treatment with anthracyclines impairs the systolic phase [37, 41, 42]. Apart from patients treated for Hodgkin lymphoma, also those with Wilms tumour located in the left kidney (radiation to the site of the removed kidney), with lung involvement and/or exposed to anthracyclines are at particular risk of cardiac complications [41, 43].

Radiation directed to the cardiac region also leads to valvular damage, and the proportion of patients with valve dysfunction increases with the time of the follow-up. Aortal stenosis is the most common disorder, as well as postradiation pericarditis, with exudative, fibrosis or chronic constricting pericarditis [37].

Damage to cerebral vessels is a substantial problem occurring after head and neck radiotherapy. Mineralizing microangiopathy is observed in patients treated for leukaemia with CNS radiation at a dose of > 20 Gy. Radiotherapy of CNS tumours or sarcomas located in the head/neck may cause atherosclerotic lesions in brain vessels, moyamoya syndrome, characterized by progressive, bilateral stenosis of cervical arteries. Changes in brain vessels promote cerebral strokes and pose a major threat to patients after CNS radiation in the course of treatment for brain tumours, leukaemia and mantle field radiotherapy in Hodgkin lymphomas [37, 43-46].

The disorders discussed above are only a few of the problems that affect survivors of childhood cancers. A number of pathologies involving other organs (kidneys, endocrine gland) also contribute to a considerable deterioration of their health condition and life quality. Multicentre and longterm observations allow us to elucidate the causes and types of organ dysfunctions in order to undertake prophylactic and therapeutic actions as well as to search for new 
directions in anticancer therapies to make them targeted, efficient and less toxic.

\section{References}

1. Mertens AC. Cause of mortality in 5-Year survivors of childhood cancer. Pediatr Blood Cancer 2007; 48: 723-6.

2. Diller L, Chow EJ, Gurney JG, et al. Chronic disease in the Childhood Cancer Survivor Study cohort: a review of published findings. J Clin Oncol 2009; 27: 2339-53.

3. Reulen RC, Winter DL, Frobisher C, et al. Long-term cause-specific mortality among survivors of childhood cancer. JAMA 2010; 304: 172-9.

4. Haupt R, Valsecchi MG, Silvestri D, De Lorenzo P, Napoli S, Masera G, Terracini B, Jankovic M. Early and late deaths after elective end of therapies for childhood cancer in Italy. Int J Cancer 2000; 86: 393-8.

5. Oeffinger KC, Mertens AC, Sklar CA, et al.; Childhood Cancer Survivor Study. Chronic health conditions in adult survivors of childhood cancer. N Engl J Med 2006; 355: 1572-82.

6. Armstrong GT, Liu Q, Yasui Y, Neglia JP, Leisenring W, Robison LL, Mertens AC. Late mortality among 5 -year survivors of childhood cancer: a summary from the Childhood Cancer Survivor Study. J Clin Oncol 2009; 27: 2328-38.

7. Mertens AC, Liu Q, Neglia JP, Wasilewski K, Leisenring W, Armstrong GT, Robison LL, Yasui Y. Cause-specific late mortality among 5-year survivors of childhood cancer: the Childhood Cancer Survivor Study. J Nat Cancer Inst 2008; 100: 1368-79.

8. MacArthur AC, Spinelli JJ, Rogers PC, Goddard KJ, Abanto ZU, McBride ML. Mortality among 5-year survivors of cancer diagnosed during childhood or adolescence in British Columbia, Canada. Pediatr Blood Cancer 2007; 48: 460-7.

9. Möller TR, Garwicz S, Barlow L, et al.; Association of the Nordic Cancer Registries; Nordic Society for Pediatric Hematology and Oncology. Decreasing late mortality among five-year survivors of cancer in childhood and adolescence: a population-based study in the Nordic countries. J Clin Oncol 2001; 19: 3173-81.

10. Ng AK, Kenney LB, Gilbert ES, Travis LB. Secondary malignancies across the age spectrum. Semin Radiat Oncol 2010; 20: 67-78.

11. Cağlar K, Varan A, Akyüz C, Selek U, Kutluk T, Yalçin B, Atahan IL, Büyükpamukçu M. Second neoplasms in pediatric patients treated for cancer: a center's 30-year experience. J Pediatr Hematol Oncol 2006; 28: 374-8.

12. Meadows AT, Friedman DL, Neglia JP, et al. Second neoplasms in survivors of childhood cancer: findings from the Childhood Cancer Survivor Study cohort. J Clin Oncol 2009; 27: 2356-62.

13. Armstrong GT, Stovall M, Robison LL. Long-Term Effects of Radiation Exposure among Adult Survivors of Childhood Cancer: Results from the Childhood Cancer Survivor Study. Radiat Res 2010; 174: 840-850

14. Oeffinger KC, Hudson MM. Long-term complications following childhood and adolescent cancer: foundations for providing risk-based health care for survivors. CA Cancer J Clin 2004; 54: 208-36.

15. Amstrong GT. Long-term survivors of childhood central nervous system malignancies: the experience of the Childhood Cancer Survivor Study. Eur J Pediatr Neurol 2010; 14: 298-303.

16. Neglia JP, Robison LL, Stovall M, et al. New primary neoplasms of the central nervous system in survivors of childhood cancer: a report from the Childhood Cancer Survivor Study. J Natl Cancer Inst 2006; 98: 1528-37.

17. Mody R, Li S, Dover DC, et al. Twenty-five-year follow-up among survivors of childhood acute lymphoblastic leukemia: a report from the Childhood Cancer Survivor Study. Blood 2008; 15: 5515-23.

18. Maule M, Scélo G, Pastore G, et al. Risk of second malignant neoplasms after childhood leukemia and lymphoma: an international study. J Natl Cancer 2007; 88: 790-800.

19. Socié G, Curtis RE, Deeg HJ, et al. New malignant diseases after allogeneic marrow transplantation for childhood acute leukemia. Clin Oncol 2000; 18: 348-57.

20. Pommier P, Sunyach MP, Pasteuris C, Frappaz D, Carrie C. Second cancer after total-body irradiation (TBI) in childhood. Strahlenther Onkol 2009; 185 suppl 2: 13-6.
21. Rihani R, Bazzeh F, Faqih N, Sultan I. Secondary hematopoietic malignancies in survivors of childhood cancer: an analysis of 111 cases from the Surveillance, Epidemiology, and End Result-9 registry. Cancer 2010; 116: 4385-94.

22. Rubino C, Adjadj E, Guérin S, et al. Long-term risk of second malignant neoplasms after neuroblastoma in childhood: role of treatment. Int J Cancer 2003; 107: 791-6.

23. Laverdiére C, Liu Q, Yasui Y, et al. Long-term outcomes in survivors of neuroblastoma: a report from the Childhood Cancer Survivor Study. J Natl Cancer Inst 2009; 101: 1131-40.

24. Bhatia S, Constine LS. Late morbidity after successful treatment of children with cancer. Cancer J 2009; 15: 174-80.

25. Lin HM, Teitell MA. Second malignancy after treatment of pediatric hodgkin disease. J Pediatr Hematol Oncol 2005; 27: 28-36.

26. Landier W, Bhatia S. Cancer survivorship: a pediatric perspective. Oncologist 2008; 13: 1181-92.

27. Henderson TO, Amsterdam A, Bhatia S, et al. Systematic review: surveillance for breast cancer in women treated with chest radiation for childhood, adolescent, or young adult cancer. Ann Intern Med 2010; 152: 444-55.

28. Taylor AJ, Croft AP, Palace AM, Winter DL, Reulen RC, Stiller CA, Stevens MC, Hawkins MM. Risk of thyroid cancer in survivors of childhood cancer: results from the British Childhood Cancer Survivor Study. Int J Cancer 2009; 125: 2400-5.

29. Bredenfeld H, Franklin J, Nogova L, et al. German Hodgkin's Lymphoma Study Group. Severe pulmonary toxicity in patients with advanced-stage Hodgkin's disease treated with a modified bleomycin, doxorubicin, cyclophosphamide, vincristine, procarbazine, prednisone, and gemcitabine (BEACOPP) regimen is probably related to the combination of gemcitabine and bleomycin: a report of the German Hodgkin's Lymphoma Study Group. J Clin Oncol 2004; 22: 2424-9.

30. Wieringa J, van Kralingen KW, Sont JK, Bresters D. Pulmonary function impairment in children following hematopoietic stem cell transplantation. Pediatr Blood Cancer 2005; 45: 318-23.

31. Oguz A, Tayfun T, Citak EC, Karadeniz C, Tatlicioglu T, Boyunaga O, Bora $\mathrm{H}$. Long-term pulmonary function in survivors of childhood Hodgkin disease and non-Hodgkin lymphoma. Pediatr Blood Cancer 2007; 49: 699-703.

32. Mertens AC, Yasui Y, Liu Y, Stovall M, Hutchinson R, Ginsberg J, Sklar C, Robison LL; Childhood Cancer Survivor Study. Pulmonary complications in survivors of childhood and adolescent cancer. A report from the Childhood Cancer Survivor Study. Cancer 2002; 95: 2431-41.

33. Carver JR, Shapiro CL, Ng A, et al. American Society of Clinical Oncology clinical evidence review on the ongoing care of adult cancer survivors: cardiac and pulmonary late effects. J Clin Oncol 2007; 25: 3991-4008.

34. Nysom K, Holm K, Olsen JH, Hertz H, Hesse B. Pulmonary function after treatment for acute lymphoblastic leukaemia in childhood. Br J Cancer 1998; 78: 21-7.

35. Yahalom J, Portlock CS. Long-term cardiac and pulmonary complications of cancer therapy. Hematol Oncol Clin North Am 2008; 22: 305-18.

36. Shankar SM, Marina N, Hudson MM, et al. Cardiovascular Disease Task Force of the Children's Oncology Group. Monitoring for cardiovascular disease in survivors of childhood cancer: report from the Cardiovascular Disease Task Force of the Children's Oncology Group. Pediatrics 2008; 121: e387-96.

37. Lipshultz SE, Lipsitz SR, Mone SM, et al. Female sex and drug dose as risk factors for late cardiotoxic effects of doxorubicin therapy for childhood cancer. N Engl J Med 1995; 332: 1738-43.

38. Lipshultz SE, Lipsitz SR, Sallan SE, Dalton VM, Mone SM, Gelber RD, Colan SD. Chronic progressive cardiac dysfunction years after doxorubicin therapy for childhood acute lymphoblastic leukemia. J Clin Oncol 2005; 23: 2629-36.

39. Lipshultz SE, Colan SD, Gelber RD, Perez-Atayde AR, Sallan SE, Sanders SP. Late cardiac effects of doxorubicin therapy for acute lymphoblastic leukemia in childhood. N Engl J Med 1991; 324: 808-15.

40. Kremer LC, van der Pal HJ, Offringa M, van Dalen EC, Voūte PA. Frequency and risk factors of subclinical cardiotoxicity after anthracycline therapy in children: a systematic review. Ann Oncol 2002; 13: 819-29. 
41. Geenen MM, Bakker PJ, Kremer LC, Kastelein JJ, van Leeuwen FE. Increased prevalence of risk factors for cardiovascular disease in long-term survivors of acute lymphoblastic leukemia and Wilms tumor treated with radiotherapy. Pediatr Blood Cancer 2010; 55 690-7.

42. Rathe M, Carlsen NL, Oxhøj H, Nielsen G. Long-term cardiac followup of children treated with anthracycline doses of $300 \mathrm{mg} / \mathrm{m} 2$ or less for acute lymphoblastic leukemia. Pediatr Blood Cancer 2010; 54: 444-8.

43. Bowers DC, Liu Y, Leisenring W, McNeil E, Stovall M, Gurney JG, Robison LL, Packer RJ, Oeffinger KC. Stroke as a late treatment effect of Hodgkin's disease: a report from the Childhood Cancer Survivor Study. J Clin Oncol 2005; 23: 6508-15.

44. Bowers DC, Liu Y, Leisenring W, et al. Late-occurring stroke among long-term survivors of childhood leukemia and brain tumors: a report from the Childhood Cancer Survivor Study. J Clin Oncol 2006; 24: 5277-82.

45. Armstrong GT, Liu Q, Yasui Y, et al. Long-term outcomes among adult survivors of childhood central nervous system malignancies in the Childhood Cancer Survivor Study. I Natl Cancer Inst 2009; 101 946-58.

46. Morris B, Partap S, Yeom K, Gibbs IC, Fisher PG, King AA. Cerebrovascular disease in childhood cancer survivors: A Children's Oncology Group Report. Neurology 2009; 73: 1906-13.

\section{Address for correspondence}

Prof. Maryna Krawczuk-Rybak MD, PhD

Department of Pediatric Oncology and Hematology

Medical University of Bialystok

Waszyngtona 17

15-274 Bialystok

e-mail: rybak@umwb.edu.pl 